\title{
Beta-Like Importins Mediate the Nuclear Translocation of MAPKs
}

\author{
Eldar Zehorai Rony Seger \\ Department of Biological Regulation, The Weizmann Institute of Science, Rehovot, Israel
}

\section{Key Words}

MAPKs $・ J N K \cdot$ p38 $・$ Nuclear translocation $・$ Beta-like importins

\begin{abstract}
Background/Aims: The rapid nuclear translocation of signaling proteins upon stimulation is important for the regulation of de-novo gene expression. However, the molecular mechanisms of this translocation is not well understood, although some studies suggest that much of this translocation may be mediated by beta-like importins (Imps). Here we undertook to study the stimulated nuclear shuttling of JNK and p38 MAPKs. Methods: For this purpose, we used coimmunoprecipitation, proximity ligation assay, gel filtration and immunostaining to examine the mechanism of nuclear translocation of these proteins. Results: We found that JNK and p38 MAPKs translocate into the nucleus in a Ran dependent, but NLS- or NTS-independent manner, unrelated to their catalytic activity. We show that this translocation involves three $\beta$-like Imps, 3, 7 and 9. Knockdown of these Imps inhibits the nuclear translocation of the MAPKs, and thereby, phosphorylation of their transcription factor targets. We further demonstrate that the translocation requires the stimulated formation of heterotrimers composed of Imp3/ Imp7/MAPK or Imp3/Imp9/MAPK. JNK1/2 and p38 $\alpha / \beta$ bind to either Imp7 or Imp9 upon stimulated post-translational modifications of the two Imps, while Imp3 joins the complex after its stimulation-induced phosphorylation. Once formed, these heterotrimers move to the nuclear envelope where Imp3 remains, while Imp7 or Imp9 escort the MAPKs into the nucleus. Conclusion: These results suggest that $\beta$-like Imps are central mediators of stimulated nuclear translocation of signaling proteins, providing a central level of regulation of the induction of cellular processes such as transcription upon stimulation.
\end{abstract}

(C) 2019 The Author(s). Published by Cell Physiol Biochem Press GmbH\&Co. KG

\section{Introduction}

Rapid and massive nuclear translocation of signaling proteins is an important step in the induction of transcription upon extracellular stimulation $[1,2]$. Despite the importance of this process, its molecular mechanism has only been elucidated for a few signaling components. The well-understood cases include signaling proteins such as NFkB [3], which utilize the classical nuclear localization signal (NLS)-mediated binding with importin- $\alpha$ $(\operatorname{Imp} \alpha)$ and $\operatorname{Imp} \beta[4]$. Another signaling protein that utilizes NLS for its nuclear translocation 
upon stimulation is ERK5 [5]. However, many other signaling proteins (e.g. ERK, MEK, RSK, PKC, PKA) translocate to the nucleus shortly after stimulation, using distinct NLS- and $\operatorname{Imp} \alpha / \beta$ - independent mechanisms. The rapid nature of the nuclear translocation, and molecular weight of the shuttling proteins $(>40 \mathrm{kDa})$ make it unlikely that their translocation is mediated by a passive diffusion. Several other stimulus-dependent signaling mechanisms for such proteins that were proposed include Imp-like activity by the shuttled protein itself (e.g. $\beta$-catenin [6]), or the use of shuttling proteins other than Imp $\alpha / \beta($ e.g. Smad4 [7]). We have previously elucidated the molecular mechanism of ERK1/2 translocation, showing that it involves a CK2-mediated phosphorylation of two Ser residues within a nuclear translocation signal (NTS) of ERK1/2 [8-10]. This phosphorylation allows interaction with Imp7, which further facilitates ERK1/2 shuttling via nuclear pores. Aside from ERK1/2, NTSlike sequences were only seen in a few other NLS-deficient translocating protein [SMAD3, MEK1 [8], and Egr1 [11]], indicating that other proteins use a distinct mechanism for their stimulated translocation.

The involvement of Imp7 in the nuclear translocation of ERK1/2 drew our attention to the group of less studied $\beta$-like Imps (Imp 2-13, also known as Karyopherin- $\beta$ s; refs [12, 13]). This group of proteins shares a $10-20 \%$ sequence similarity amongst themselves as well as with Imp $\beta$. As in case of Imp $\beta$, they all contain helical HEAT repeats, and bind weakly to phenylalanine-glycine-repeats in the nuclear pore complex (NPC), required for directed cargo translocation. Finally, these Imps are regulated by the small GTPase Ran, which determines the directionality of the transport [14]. Most of these Imps are ubiquitously expressed, and have been shown to catalyze nuclear shuttling of both housekeeping and regulatory proteins. Among the regulatory proteins, c-Jun uses Imps $\beta, 2,5,7,9$, and 13 for its nuclear translocation in non-stimulated or stimulated cells [15], SMAD4 uses Imp7 and Imp8 [7], and HIF1, Imp4 and Imp7 [16]. The translocation of these proteins, as well as of ERK1/2, and MEK1/2 led us to hypothesize that these Imps play a general role in the stimulated nuclear translocation of signaling proteins. Although the mechanism of action of these proteins is not fully understood, they may operate by more than one way to regulate the localization of the stimulated or housekeeping proteins [12].

In a search for NLS-independent shuttling proteins, we have studied the MAPK family members JNK and p38 [17]. In mammals, there are three JNK isoforms (JNK1-3) and four p38 ones $(\mathrm{p} 38 \alpha-\delta)$ that regulate a large number of stimulated cellular processes, including primerily stress responses. Unlike ERK1/2 and ERK5 [18], the subcellular localization of JNKs and p38s has not been properly established thus far. Similarly to ERK1/2, JNK1/2 and p38 $\alpha / \beta$, interact with cytosolic anchoring proteins in resting cells $[19,20]$, and translocate to the nucleus upon stimulation. Indeed, such a process was demonstrated for both JNK1/2 $[21,22]$ and $p 38 \alpha / \beta[23-25]$ in some systems. However, in others it was suggested that JNK1/2 $[26,27]$ and $p 38 \alpha / \beta[28,29]$ are localized in the nucleus of resting cells, which may be responsible for some constant activities such as genome stability for JNK [30] and ROS production for p38 [31]. For the latter, it was even shown that after stimulation, it is exported out of the nucleus [32]. These conflicting data may indicate that the localization of these MAPKs is cell-type specific, but this still requires clarification.

In this study, we show that similar to ERK1/2, JNK1/2 and $p 38 \alpha / \beta$ are localized primarily in the cytoplasm of resting cells, and translocate to the nucleus upon stimulation. We further found that despite the pronounced similarity amongst the MAPKs, none of the JNK or p38 isoforms contain an ERK-NTS. Moreover, mutations in the equivalent regions did not have any effect on JNK1/2 or p38 $\alpha / \beta$ translocation, which occurs independent of their activation. We found that the translocation of these MAPKs is mediated by the formation of either Imp3/7/MAPK or Imp3/9/MAPK heterotrimers. This heterotrimerization occurs upon stimulation by phosphorylation of Imp3, as well as by the binding of JNK1/2 and p38 $\alpha / \beta$ to either Imp7 or Imp9. These heterotrimers then escort the MAPKs to the nuclear envelope, where Imp3 remains, whereas Imp7 or Imp9 enter the nucleus with the MAPK in a Ran-dependent manner. Thus, the stimulated nuclear translocation of MAPKs is mediated 


\section{Cellular Physiology Cell Physiol Biochem 2019;52:802-821 \\ \begin{tabular}{c|c|c|c|} 
Dol: 10.33594/000000056 & O 2019 The Author(s). Published by \\
Cell Physiol Biochem Press GmbH\&Co
\end{tabular} \\ Zehorai et al.: JNK/p38 Translocation is Mediated by Importins3, 7 and 9}

by distinct $\beta$-like Imps, demonstrating their role in the regulation of transcription and cell fate.

\section{Materials and Methods}

\section{Reagents}

Tetradecanoyl phorbol acetate (TPA), anisomicin, Polyethylenimine (PEI) and 4'6-diamino-2phenylindole (DAPI) were obtained from Sigma (Rehovot, Israel). A/G beads were purchased from Santa Cruz Biotechnology (Dallas, TX, USA). Albumin bovine serum (BSA) was purchased from MP niomedical (Solon, OH, USA). Si RNAs and Dharmafect were from Thermo Fisher Scientific (CO, USA). Proximity ligation assay kit was from Olink Bioscience (Uppsala, Sweden). NBT/BCIP developing substrates were purchased from Promega (WI, USA), Calf Intestinal Phosphatase (CIP) was from New England Biolabs (MA, USA).

\section{Antibodies}

Anti Imp2, Imp3, Imp5, Imp7 Abs were from Abnova (Taipei, Taiwan). Anti Imp9, Imp13, pMEF2a Abs were from Novus (Littleton, USA). Anti Imp4 and Imp12 Abs were from Abcam (Cambridge, England). Anti GFP Ab was from Roche Diagnostics GmbH (Mannheim, Germany). Anti Imp8, Imp10, Imp11, JNK1, JNK2, p38ß, pcMyc, Tubulin, GST and pATF2 Abs were from Santa Cruz Biotechnology (CA, USA). Abs against doubly phosphorylated ERK1/2 (pTEY-ERK), general ERK (gERK), doubly phosphorylated JNK (pJNK), general JNK1/2 (gJNK), doubly phosphorylated p38 (pp38), general p38 and c-Myc and Imp $\beta$ were obtained from Sigma Israel (Rehovot, Israel). Anti pC-Jun, JNK2 and p38 $\alpha$ were from Cell Signaling Technology (Boston, MA, USA). All antibodies were specific and recognize just one band in Western blot under the conditions used. Secondary antibodies conjugated to HRP or AP as well as secondary light chain specific secondary antibodies were purchased from Jackson Immunoresearch (West Grove, PA, USA).

\section{Cell Culture and transfection}

HeLa and MCF7 cells were cultured in Dulbecco's modified Eagle's medium (DMEM) supplemented with $2 \mathrm{mM}$ L-glutamine, 1\% Pen/Strep and 10\% fetal bovine serum (FBS). HB2 cells were cultured in the same medium with the addition of hydrocortisone $(0.5 \mathrm{mg} / \mathrm{ml})$, and insulin $(10 \mu \mathrm{g} / \mathrm{ml})$. MCF10A cells were cultured in DMEM / F-12 with 5\% horse serum, EGF $(200 \mathrm{ng} / \mathrm{ml})$, hydrocortisone $(0.5 \mathrm{mg} / \mathrm{ml})$, cholera toxin $(100 \mathrm{ng} / \mathrm{ml})$, insulin $(10 \mu \mathrm{g} / \mathrm{ml}), 2 \mathrm{mM}$ L-glutamine, $1 \%$ pen/strep and $10 \% \mathrm{FBS}$. HeLa cells were transfected using polyethylenimine (Sigma, [33]). SiRNAs were transfected using Dharmafect (Thermo Fisher Scientific, CO, USA).

\section{Immunofluorescence microscopy}

Cells were fixed in $3 \%$ paraformaldehyde in PBS $\left(20 \mathrm{~min}, 23^{\circ} \mathrm{C}\right)$, and incubated with $2 \%$ Albumin bovine serum (BSA) in PBS $\left(15 \mathrm{~min}, 23^{\circ} \mathrm{C}\right)$, followed by permeabilization with Triton $\mathrm{X}-100(0.1 \%$ in PBS, $\left.5 \mathrm{~min}, 23^{\circ} \mathrm{C}\right)$. Cells were incubated with the primary Abs $\left(60 \mathrm{~min}, 23^{\circ} \mathrm{C}\right)$, washed three times with PBS and incubated with rhodamine-conjugated secondary $\mathrm{Ab}\left(60 \mathrm{~min}, 23^{\circ} \mathrm{C}\right)$, and DAPI. Slides were visualized by either fluorescence microscope (Olympus BX51, x40 magnification), or spinning disk confocal microscope (Zeiss, Cell observer SD, x100 magnification). Background correction, and contrast adjustment of raw data were performed using Photoshop (Adobe, CA, USA).

\section{DNA Constructs and mutations}

GFP-JNK1/2, p38 $\alpha / \beta$ were cloned in pEGFP-C1 (Clontech, Mountain View, CA). JNK1/2 and p38 $\alpha / \beta$ sequences were amplified from HeLa cells cDNA and flanked by EcoRI / BamHI for JNK2 and p38 $\beta$ and with XhoI / BamHI for JNK1 and p38 $\alpha$. Point mutations of JNK1/2 and p38 $\alpha / \beta$ were performed by site-directed mutagenesis. GST-JNK1/2, p38 $\alpha / \beta$ were cloned in pGEX-2T vector (GE healthcare, Buckinghamshire, UK) and flanked by SpeI/NotI restriction sites. Imps 3, 7 and 9 were cloned in pEGFP-C. Imps 7 and 9 were amplified from HeLa cells cDNA using specific primers flanked by BamHI/Sall for Imp7 and XhoI/SalI for Imp9, restriction sites. Imp3 was acquired from Forchheimer repository plasmid collection, and amplified using specific primers flanked by XhoI/EcoRI restriction sites. GST-Imp3, GST-Imp9 and His-Imp7 were a generous gift from Prof. Oded Livnah (Hebrew Univercity, Jeruslaem, Israel). 


\section{Cellular Physiology Cell Physiol Biochem 2019;52:802-821 \\ \begin{tabular}{c|c|c|} 
Dol: 10.33594/000000056 & O 2019 The Author(s). Published by \\
Cell Physiol Biochem Press GmbHerco
\end{tabular} \\ Zehorai et al.: JNK/p38 Translocation is Mediated by Importins3, 7 and 9}

\section{Coimmunoprecipitation (CoIP)}

CoIP was done as previously descibed ([8]. Briefly, cell extracts were produced and incubated for $2 \mathrm{hr}$ $\left(4^{\circ} \mathrm{C}\right.$, with rotation) with $\mathrm{A} / \mathrm{G}$-agarose beads pre-linked with specific $\mathrm{Abs}\left(1 \mathrm{hr}, 23^{\circ} \mathrm{C}\right)$. The bound $\mathrm{A} / \mathrm{G}$ beads were washed, resuspended (1.5X sample buffer), boiled and subjected to Western blotting.

\section{Proximity ligation assay (PLA)}

Protein-protein interactions were detected with Duolink PLA Kit ([34]; Olink Bioscience), according to the manufacturer's protocol. Briefly, cells were grown, fixed and permeabilized as described. The samples were then incubated with primary Abs against two examined proteins $\left(60 \mathrm{~min}, 23^{\circ} \mathrm{C}\right)$, washed (0.01 M Tris $\mathrm{HCl} \mathrm{pH} \mathrm{7.4,} 0.15 \mathrm{M} \mathrm{NaCl}$ and $0.05 \%$ Tween 20), and then incubated with specific probes (60 min, $\left.37^{\circ} \mathrm{C}\right)$, following by DAPI staining to visualize nuclei and washed $(0.2 \mathrm{M}$ Tris $\mathrm{HCl} \mathrm{pH} \mathrm{7.5,} 0.15 \mathrm{M} \mathrm{NaCl})$. The signal was visualized as distinct fluorescent spots by a fluorescence microscope (Olympus BX51, x40 magnification). Background correction, contrast adjustment and the quantification of the fluorescence signal were performed using Photoshop and ImageJ.

\section{In vitro interaction assay}

Cell extracts were produced as described and incubated for $2 \mathrm{hr}\left(4^{\circ} \mathrm{C}\right)$ with $\mathrm{A} / \mathrm{G}$-agarose beads prelinked with specific Abs $\left(1 \mathrm{hr}, 23^{\circ} \mathrm{C}\right)$. The bound $\mathrm{A} / \mathrm{G}$ beads were sequentially washed with RadioImmune Precipitation Assay (RIPA) buffer, twice with $0.5 \mathrm{M} \mathrm{LiCl}$ and twice with Buffer A (50 mM $\beta$-glycerophosphate pH 7.3, $1.5 \mathrm{mM}$ EGTA, $1 \mathrm{mM}$ EDTA, $1 \mathrm{mM}$ dithiothreitol, and $0.1 \mathrm{mM}$ sodium vanadate). The bound A/G beads were then resuspended in Buffer A containing $0.01 \%$ BSA and aliquoted. GST tagged proteins were incubated with the beads $2 \mathrm{hr}\left(4^{\circ} \mathrm{C}\right.$, with rotation) then washed and resuspended in $1.5 \mathrm{X}$ sample buffer and boiled.

\section{In vitro phosphatase assay}

HeLa cells were grown to subconfluency, serum-starved ( $0.1 \%$ FBS for $16 \mathrm{hr})$ and then treated with stimuli or other drugs. Cell extracts were produced as described and incubated for $2 \mathrm{hr}\left(4^{\circ} \mathrm{C}\right.$, with rotation) with A/G-agarose beads (Santa Cruz Biotechnology) pre-linked with specific Abs $\left(1 \mathrm{hr}, 23^{\circ} \mathrm{C}\right)$. The bound $\mathrm{A} / \mathrm{G}$ beads were sequentially washed once with RIPA buffer, then twice with $0.5 \mathrm{M} \mathrm{LiCl}$ and twice with Buffer A. The bound A/G bead sample, originated from the stimulated cells, was then incubated with Calf Intestinal Phosphatase $\left(37^{\circ} \mathrm{C}, 1 \mathrm{hr}, \mathrm{NEB}, \mathrm{MA}, \mathrm{USA}\right)$ then washed. The samples were then resuspended in Buffer A containing $0.01 \% \mathrm{BSA}$ and aliquoted-GST tagged proteins were incubated with beads $\left(4^{\circ} \mathrm{C}, 2 \mathrm{hr}\right)$, then washed and resuspended in sample buffer and boiled.

\section{Subcellular Fractionation}

Subcellular fractionation was performed essentially as described [8]. Briffly, Harvested cells were then resuspended in $200 \mu \mathrm{l}$ of buffer $\mathrm{H}$ containing $0.1 \%$ Nonidet P- 40 . The lysates were mixed vigorously and centrifuged immediately as above to yield supernatants containing the cytosolic fraction. Nuclear proteins were extracted by resuspending the nuclear pellets in $200 \mu \mathrm{l}$ of extraction buffer, waiting on ice for $5 \mathrm{~min}$, brief sonication $\left(2 \times 5 \mathrm{sec}, 40 \mathrm{~W}, 4^{\circ} \mathrm{C}\right)$, vigorous mixing, and centrifugation. The protein concentration was determined by Coomassie protein assay reagent (Pierce). Both cytosolic and nuclear fractions were subjected to Western blotting.

\section{Gel filtration}

HeLa cells were serum starved (as above) and then were either stimulated with anisomycin (Anis, 0.5 $\mu \mathrm{g} / \mathrm{ml}, 15 \mathrm{~min}$ ) and TPA (250 $\mathrm{nM}, 15 \mathrm{~min}$ ), or left untreated (NT). Cell extracts (25 mg of each treatment) were loaded on a 16/60 superdex 200 sizing column (flow rate $1 \mathrm{ml} / \mathrm{min}$ ), and $1 \mathrm{ml}$ fractions were collected. The fractions were analyzed using Western blot with the appropriate Abs. 


\section{Cellular Physiology Cell Physiol Biochem 2019;52:802-821 \\ \begin{tabular}{ll|l} 
and Biochemisty $10.33594 / 000000056$ & (c) 2019 The Author(s). Published by \\
Cell Physiol Biochem Press GmbH\&Co. KG
\end{tabular} \\ Zehorai et al.: JNK/p38 Translocation is Mediated by Importins3, 7 and 9}

\section{Statistical Analysis}

Data are expressed as mean \pm S.E. Statistical evaluation was carried out using functional analysis and Student's t test (two-tailed) to test for differences between the control and experimental results. Values of $p<0.05$ were considered statistically significant. In Fig. 1, 2, 3, MAPK staining that was either all over or primarily in the nucleus (more than $40 \%$ nuclear staining, no darker nuclear staining) was considered nuclear.

\section{Results}

JNK and p38 translocate into the nucleus using a NTS-independent mechanism

In our search for NLS-independent shuttling proteins, we have looked into the subcellular localization of JNK1/2 and $\mathrm{p} 38 \alpha / \beta$. Using immunostaining with specific antibodies (Abs) we found that, similar to ERK1/2, JNK1/2 and $\mathrm{p} 38 \alpha / \beta$ are localized mainly in the cytoplasm of resting cells (Fig. 1A). Treating the cells with either stress or mitogenic stimulants (anisomycin and TPA, respectively), we observed a rapid and rubost nuclear translocation of all four MAPK isoforms, with only minor differences in their kinetics of translocation. This translocation of JNKs and p38s was confirmed using subcellular fractionation of anisomycintreated HeLa cells (Fig. 1B). Interestingly, this translocation did not correlate with JNK1/2 and $\mathrm{p} 38 \alpha / \beta$ activatory phosphorylation (Fig. 1C). Both JNK and p38 peak at 30 min after stimulation with anisomycin (fold activation avarages from three distinct experiments were $13.5 \pm 1.5$ fold for JNK and 9.6 \pm 2.2 fold for $\mathrm{p} 38$ ), while despite of the significant translocation, no activation was detected with TPA. These results indicate that the phosphorylation of JNK and p38 is not required for the translocation and that the mechanism of translocation of these four MAPKs is different from that of ERK1/2. Moreover, none of the JNK or p38 isoforms contained an NTS, and only two, JNK2 and p38 $\beta$ contained a phosphorylatable sequence (T-P-S) in the same kinase region (Fig. 2A). Nonetheless, the pronounced sequence and conformation similarities among JNK1/2 and p38 $\alpha / \beta$ to ERK1/2 [17] prompted us to examine whether this region can act as an NTS in JNK1/2 or $\mathrm{p} 38 \alpha / \beta$ as well. For this reason, we mutated the phosphorylatable ERK-NTS-aligned residues in JNK1/2 or $\mathrm{p} 38 \alpha / \beta$ to either Ala or Glu residues in all four MAPKs. Unlike ERK1/2, overexpression of these mutants resulted in a similar distribution to that of WT proteins (Fig. 2B), indicating that JNK1/2 and p38 $\alpha / \beta$ differ from ERK1/2 not only in the release from anchoring proteins $[19,20]$, but also in their NTS, and probably the whole Imp7-dependent mechanism of ERK translocation [8].

\section{JNK1/2 and $p 38 \alpha / \beta$ interact with Imps 3, 7 and 9 upon stimulation}

The lack of ERK1/2-like NTS, as well as a canonical or any atypical NLSs [35-37] prompted the search for their actual mechanism of translocation. To detect whether the process is energy or Ran-dependent, we knockdown Ran expression, and found that it is strongly involved in the stimulated nuclear translocation of JNK and p38 (Fig. 3). These results indicate that this translocation is not mediated by simple diffusion, but requires Ran-dependent transport mechanism. Since $\beta$-like Imps are Ran-dependent and have been implicated in the stimulated translocation of signaling proteins $[12,13]$, we hypothesised that one or more of these Imps are involved in this process. To examine it, we first used a CoIP screen to detect whether any of them could interact with JNK1/2 and p38 $\alpha / \beta$. Despite the expression of all these Imps in the examined cells, no significant Imp/MAPK interactions were detected in resting HeLa cells (Fig. 4A). However, IP of JNK1/2 and p38 $\alpha / \beta$ in extracts of stimulated HeLa cells, revealed a varying degree of interaction of all four MAPKs with Imps 3, 7 and 9. Importantly, the screen and other IP experiements demonstrated only small differences in the association and timing of interactions between the components, suggesting a similar mode of regulation among them.

In addition, we performed a proximity ligation assay (PLA), which is a CoIP-independent tool for protein-protein interaction studies (See Material and Methods). Similar to the CoIP results, no significant basal interactions between the examined Imps and MAPKs were 


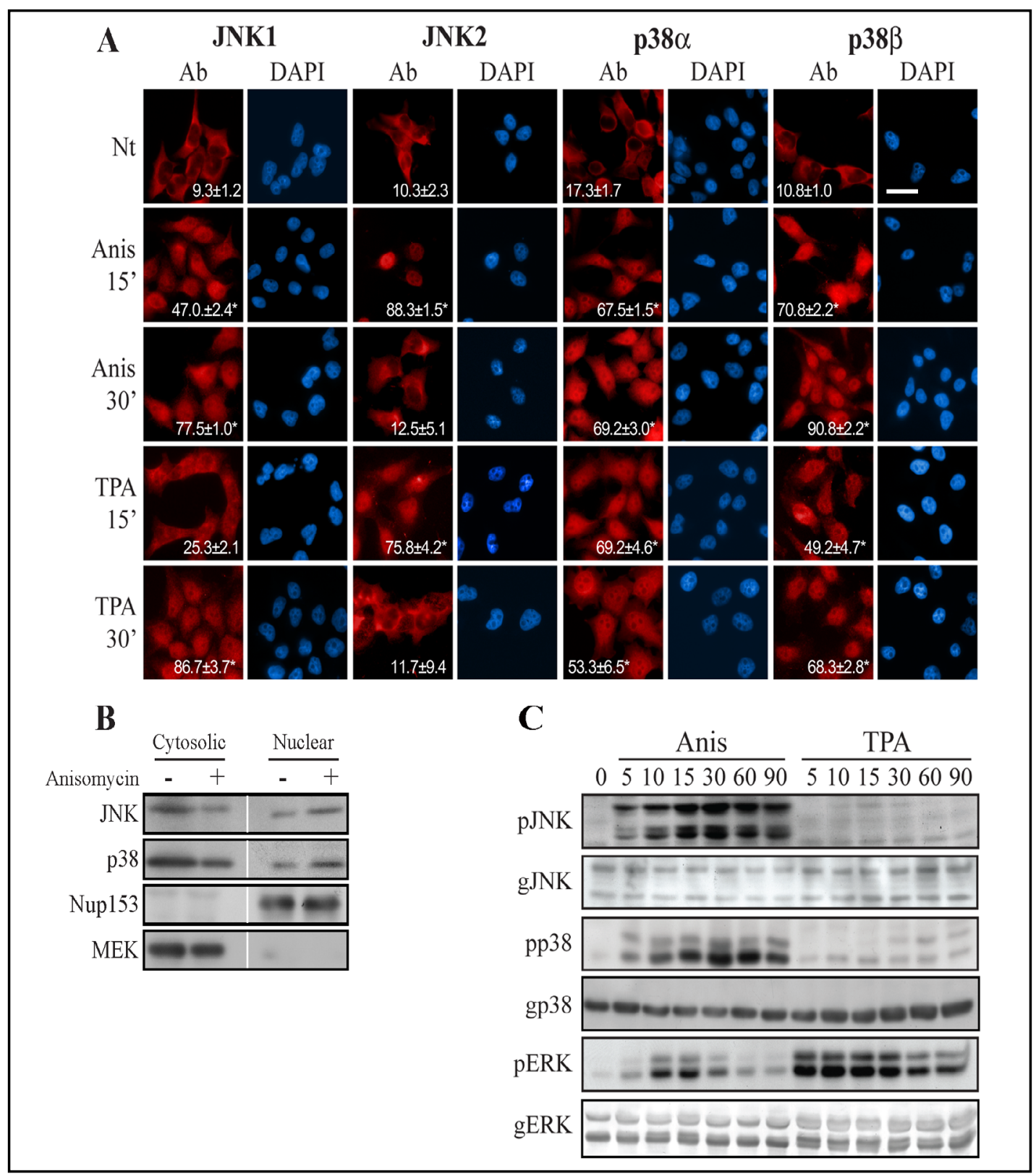

Fig. 1. JNK1/2 and $\mathrm{p} 38 \alpha / \beta$ translocate into the nucleus independently of their activatory phosphorylation. (A) Fluorescent microscopy demonstrate the stimulated nuclear translocation of JNK1/2 and p38 $\alpha / \beta$. HeLa cells were grown on slides to $70 \%$ confluence, serum starved $(0.1 \% \mathrm{FBS}, 16 \mathrm{hr})$, and then stimulated with anisomycin (Anis, $0.5 \mu \mathrm{g} / \mathrm{ml}$ ) or TPA $(250 \mathrm{nM})$ for the indicated times, or left untreated. Cells were fixed, and stained with anti JNK1, JNK2, p38 $\alpha$ or p38 $\beta$ Abs as indicated, and DAPI to detect nuclei. Percent cells with $>40 \%$ nuclear staining and standard error was determenined by counting six distinct fields of 20 cells each from two distinct experiments. ${ }^{*}$ - $\mathrm{P}<0.05$ compaired to non-stimulated cells. The bar in the upper right panel is of $20 \mu \mathrm{M}$. (B) Subcellular fractionation confirms the nuclear translocation of JNK1/2 and p38 $\alpha / \beta$. Serum-starved HeLa cells were either stimulated (Anis, $0.5 \mu \mathrm{g} / \mathrm{ml}, 15 \mathrm{~min}$ ) or left untreated, and then harvested. Subcellular fractions of cytosolic and nuclear were produced as described under materials and methods, and the fractions were subjected to Western blot analysis with the indicated Abs. (C) The nuclear translocation of endogenous JNK1/2 and $\mathrm{p} 38 \alpha / \beta$ is independent of their phosphorylation. HeLa cells were grown to $70 \%$ confluence, serum starved, and then stimulated for the indicated time points. Cell extracts were produced and subjected to Western blot analysis with the indicated Abs. The blots were developed with NBT/BCIP or ECL. The experiments were reproduced with very similar results three times. 


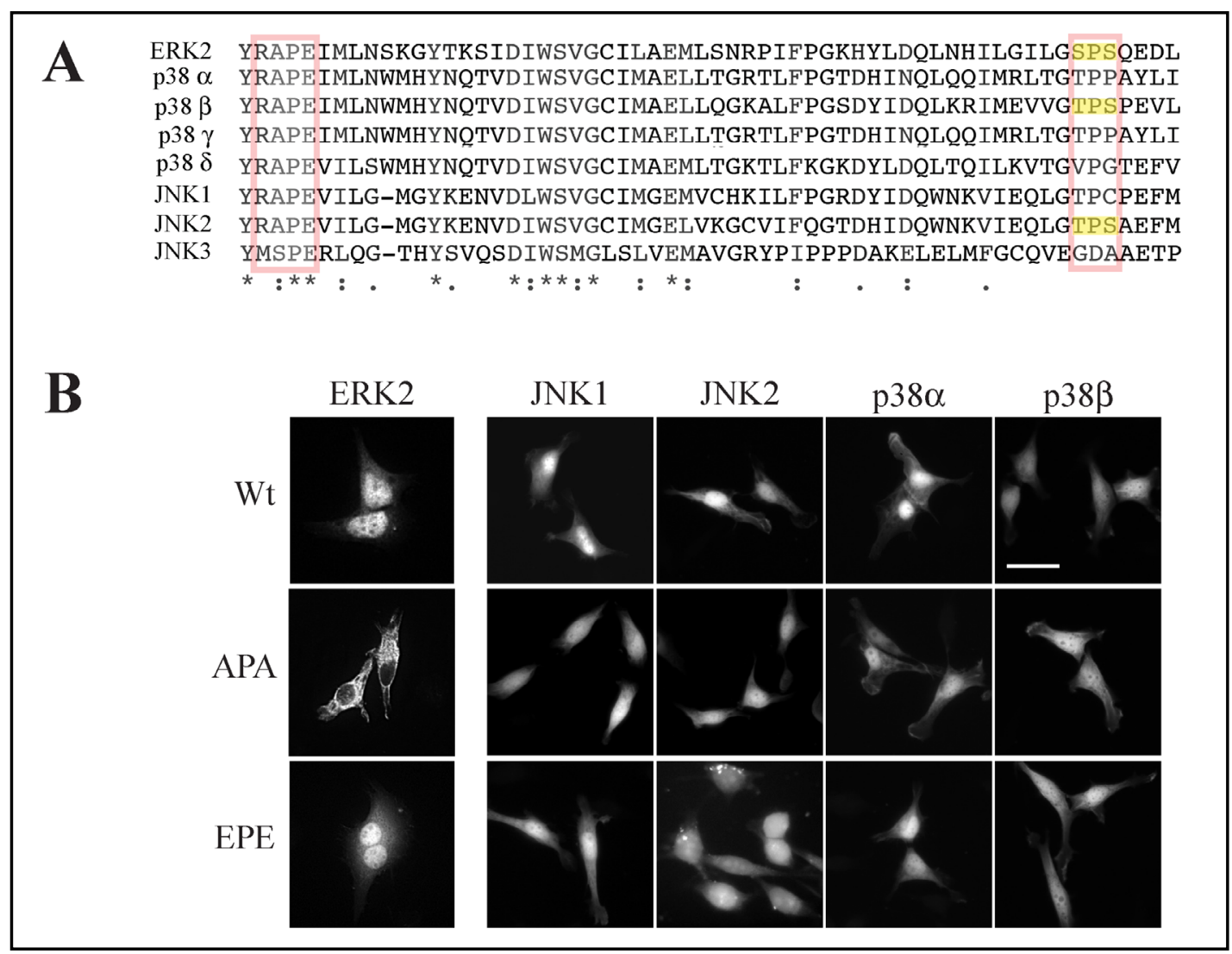

Fig. 2. JNK1/2 and $\mathrm{p} 38 \alpha / \beta$ translocate into the nucleus using NTS-independent mechanism. (A) Sequence alignment of the kinase insert domain (KID) and the NTS in ERK2 compared with JNKs/p38s. The left side box mark the conserved kinase residues of the kinase subdomain VIII, that are adjacent to the KID. The right side box mark the region of ERK1/2's-NTS (SPS motif). The phosphorylatable residues in this region are marked in yellow. (B) Mutations in the NTS-homologous region do not affect the nuclear shuttling of JNK1/2 and $\mathrm{p} 38 \alpha / \beta$. HeLa cells were grown on slides to $70 \%$ confluence, transfected with JNK1-GFP (wt-JNK1), JNK2-GFP (wt-JNK2), p38 $\alpha$-GFP (wt-p38 $\alpha$ ) or p38 $\beta$-GFP (wt-p38 $)$ ) and their mutants (APA or EPE). Sixteen $\mathrm{hr}$ after transfection the cells were serum starved, fixed and visualized using a fluorescent microscope (Olympus BX51, x40 magnification). The results were reproducible and similar localization was observed in $>50$ cells for each condition. The bar in the upper right panel is of $20 \mu \mathrm{M}$.

detected (Fig. 4B). However, anisomycin or TPA stimulation resulted in a marked increase in the interaction of either JNK1/2 or p38 $\alpha / \beta$ with Imps 3,7 and 9 . As would be expected from the shuttling role of the Imps, the interactions were found mostly in the cytoplasm and perinuclear regions. To verify these interactions, we repeated the CoIP experiments in MCF7 cells, and examined the interactions of overexpressed MAPKs with the Imps in HeLa cells. Both experiments confirmed that the interaction of MAPKs with of Imps 3, 7 and 9 is not specific only to endogenous HeLa proteins (Fig. 5). Moreover, we performed CoIP experiments using overexpressed MAPKs and found that in most cases they interact with Imp3, 7 and 9 as well. There were two reproducible exceptions to this type of interaction (JNK2 and Imp3 and $\mathrm{p} 38 \beta$ and Imp9 upon aniscomycin treatment). The reason for these changes is not clear at this stage, but might occur due to eronious localization or regulation of the overexpressed proteins. Together, our results may indicate that the stimulated interaction of Imps 3, 7 and 9 with the MAPKs first occurs in the cytoplasm, and the Imps are detached from the MAPKs either during the shuttling, or immediately after translocation. 


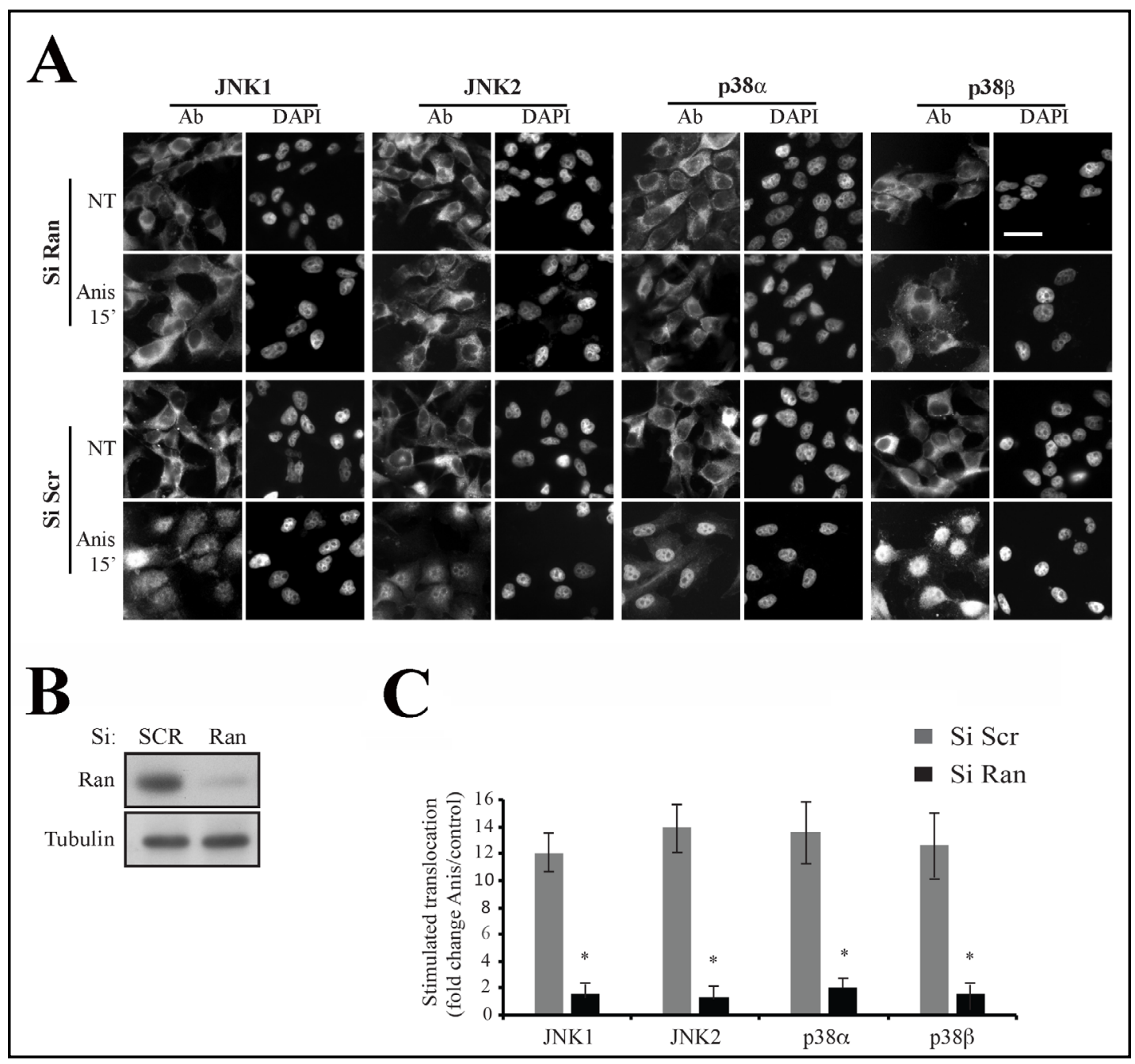

Fig. 3. Ran is required for JNK1/2 and $\mathrm{p} 38 \alpha / \beta$ nuclear translocation. HeLa cells were grown on cover slides to $30 \%$ confluence. SiRNA of Ran and a scrambled siRNA (SiScr; serves as a negative control) were then transfected to the cells using Dharmafect according to the manufacturer's instructions. Forty-eight hr after transfection, the cells were serum starved, and then either stimulated with anisomycin (anis; $0.5 \mu \mathrm{g} / \mathrm{ml}, 15$ $\mathrm{min}$ ), or left untreated (NT) as indicated. The cells were then fixed, stained using the indicated anti MAPK Abs and visualized using a fluorescent microscope. The bar in the upper right panel is of $20 \mu \mathrm{M}$. B. SiRNA reduces the amount of Ran. To confirm the efficiency of the siRNA, cell were transfected with siRNAs as described, and were subjected to a Western blot analysis with the indicated Abs (lower panel). C. Quanntification. The results in A were quantified (total of 60 cells in 4 fields from two distinct experiment. The fold change in cells with nuclear staining ( $>40 \%$ ) upon anisomycin stimulation was calculated in cells treated with anisomycin in both control Si Scr and Si Ran. * $\mathrm{P}<0.01$ between Anis treated and untreated cells.

\section{Imps 3, 7 and 9 are required for JNK1/2 or $p 38 \alpha / \beta$ translocation into the nucleus}

To further substantiate the role of Imps 3, 7, and 9 in the nuclear translocation of JNK1/2 or $\mathrm{p} 38 \alpha / \beta$, we used their siRNA to determine if the knockdown of these Imps affect the nuclear accumulation of MAPKs. A knockdown of Imp5, and scrambled siRNA of Imp3 served as negative controls. The siRNAs of the four Imps examined reduced the amount of the relevant Imps by more than $85 \%$ within $48 \mathrm{hr}$ (Fig. 6). These knockdowns did not affect the cytoplasmic localization of endogenous JNK1/2 or p38 $\alpha / \beta$ in resting cells (data not shown). However, the knockdown of Imps 3, 7, and 9, but not Imp5 or siRNA control, strongly modulated the stimulated nuclear shuttling of the examined MAPKs. The knockdown of Imp3 inhibited the translocation in $\sim 80 \%$ of the cells, whereas the siRNA of Imp7 and Imp9 


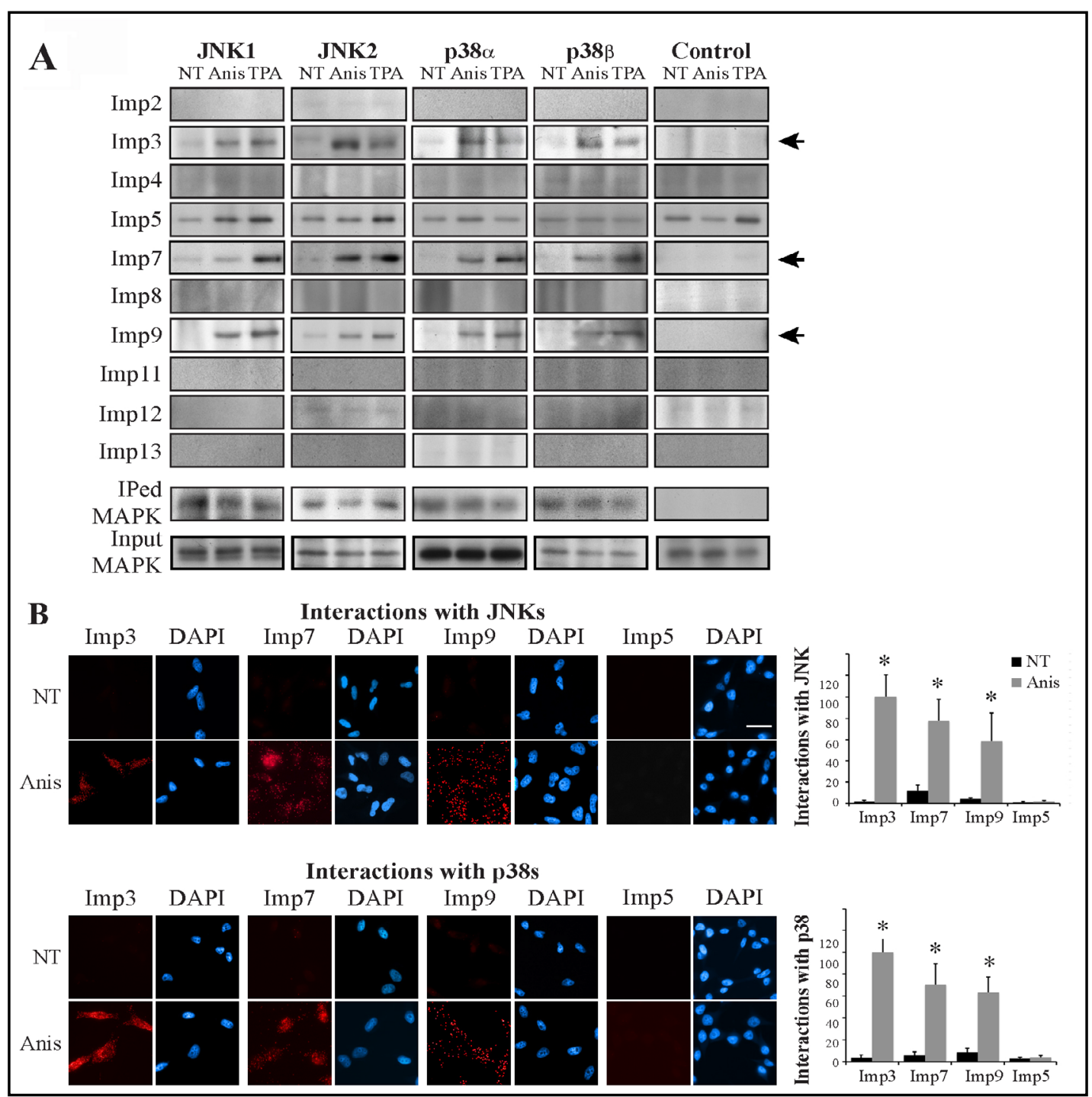

Fig. 4. JNK1/2 and p38 $\alpha / \beta$ interact with Imps 3, 7 and 9 in HeLa cells. (A) A CoIP screen to detect Imp/ MAPK interactions. HeLa cells were grown to $70 \%$ confluence, serum starved $(0.1 \% \mathrm{FBS}, 16 \mathrm{hr})$, and then stimulated with anisomycin (Anis, $0.5 \mu \mathrm{g} / \mathrm{ml}, 15 \mathrm{~min}$ ), TPA (250 nM, $15 \mathrm{~min}$ ) or left untreated (NT). Cell extracts were subjected to CoIP with anti JNK1, JNK2, p38 $\alpha$, p38 $\beta$ Abs, or rabbit IgG as a negative control (Control). The interacting Imps and amount of IPed or input MAPKs (in extracts) were detected by Western blotting with the indicated Abs (bottom panel). Arrows indicate the interacting Imps. (B) Proximity ligation assay confirms interaction of JNKs/p38s with Imps 3, 7 and 9. HeLa cells were grown on slides to 70\% confluence, serum starved and then either stimulated or left untreated (NT). Cells were fixed, and subjected to a PLA assay according to the manufacturer's instructions using the anti Imps together with either anti JNK (upper panels) or p38 (lower panels) Abs. The nuclei were detected using DAPI. Slides were visualized using a fluorescent microscope. The bar in the upper right panel is of $20 \mu \mathrm{M}$. Quantification of the intensity of the signal of 3 experiments (Bar graphs at right) was performed using Image $\left(^{*}-\mathrm{p}<0.0001\right)$.

prevented the translocation in only $\sim 60 \%$. Importantly, the combined knockdown of Imp7 and 9 significantly increased the effect of the individual ones to $~ 90 \%$ of the cells, indicating that these two Imps act additively in translocating stimulated MAPKs to the nucleus.

To further study the effect of Imps 3, 7 and 9 knockdown on the translocation of JNK1/2 or $\mathrm{p} 38 \alpha / \beta$, we examined the phosphorylation of the nuclear JNK/p38 substrates c-Jun, ATF2 and MEF2A [1]. Importantly, all three siRNAs significantly inhibited (>90\%) the stimuli- 


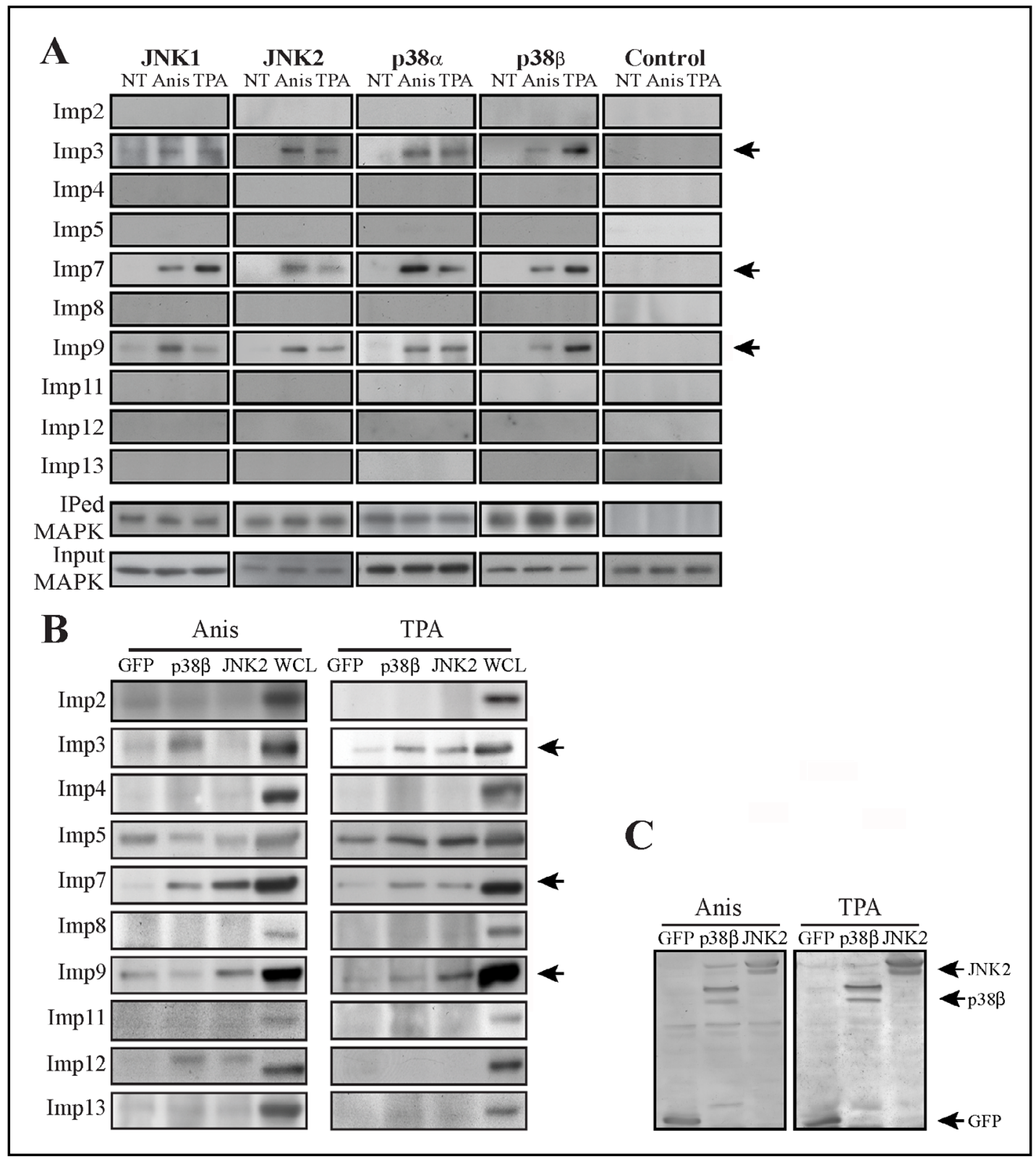

Fig. 5. Endogenous and overexpressed JNKs and p38s CoIP with Imps 3, 7 and 9. (A) A CoIP screen to detect the Imps interacting with JNK1/2 and p38 $\alpha / \beta$ in MCF7 cells. MCF7 cells were grown to $70 \%$ confluence, serums starved and then were stimulated with either anisomycin (Anis $0.5 \mu \mathrm{g} / \mathrm{ml}, 15 \mathrm{~min}$ ), TPA (250 nM, $15 \mathrm{~min}$ ) or left untreated (NT). Cells extracts were then subjected to CoIP with the indicated Abs. Imps, IPed MAPKs and inputs (in extracts) were detected by Western blot with the indicated Abs. (B) Interaction of overexpressed MAPKs with Imps 3,7 and 9 in HeLa cells. HeLa cells were transfected with either JNK2-GFP (wt-JNK2), p38 $\beta$-GFP (wt-p38 $\beta$ ) or GFP alone. Cells were serum starved, and then treated as described and subjected to CoIP with anti GFP Abs. The interacting Imps and the loading extracts were detected using Western blot analysis with the indicated Abs. Arrows indicate the interacting Imps. WCL is whole cell extract. (C) Loading control of overexpressed JNK2 and p38 $\alpha$. Extracts of HeLa cells overexpressing either JNK2-GFP or p38 $\alpha$-GFP or GFP alone were subjected to Western blot analysis with anti GFP Abs. The experiments were reproduced twice. The antibodies detected mainly the two bands (two start-sites) of the GFP-MAPKs and also few minor degradation products/impurities. 


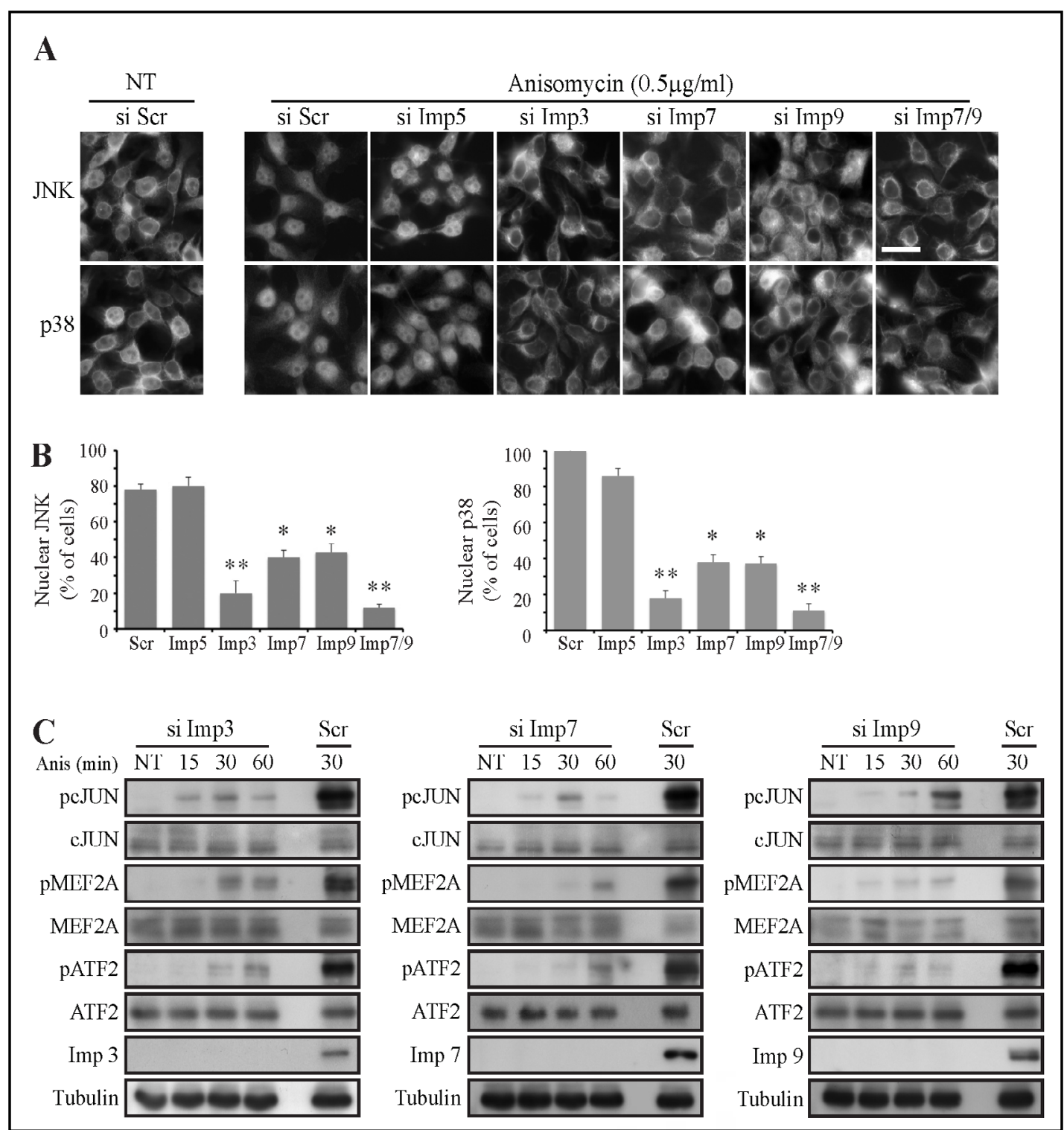

Fig. 6. Imps 3, 7 and 9 are required for JNK and p38 translocation and phosphorylation of downstream nuclear transcription factors. (A) siRNA of Imp3, 7, 9 but not of Imp5 inhibits the stimulated-nuclear translocation of JNKs and p38s. HeLa cells were grown on cover slides to 30\% confluence. Si RNAs of the indicated Imps and siRNA control (scambled siRNA of Imp3; si Scr) were transfected to the cells. $48 \mathrm{hr}$ after transfection, cells were serum starved, stimulated or left untreated (NT), fixed and stained using the indicated anti MAPK Abs. The bar in the upper right panel is of $20 \mu \mathrm{M}$. (B) Quantification of JNKs/p38s nuclear localization prior and upon stimulation. The nuclear localization of JNKs and p38s was calculated as the number of cells presenting nuclear staining (nuclear staining was considered for all cells in which > $30 \%$ of the stain was inside the nucleus) per the total cell number. The data shown represents mean \pm S.E of three different experiments ( $\left.{ }^{*}-\mathrm{p}<0.0003,{ }^{* *}-\mathrm{p}<0.00001\right)$. (C) SiRNA of Imp3, 7, 9 inhibits the induction of transcription regulated by JNKs/p38s. HeLa cells were transfected with the indicated siRNAs or control of scrambled siRNA of Imp3 (Scr), serum starved and then either stimulated with anisomycin (Anis $0.5 \mu \mathrm{g} / \mathrm{ml}$ ) for the indicated times, or left untreated (NT). Cell extracts were subjected to a Western blot analysis with the indicated Abs. The experiments were reproduced twice. 


\section{Cellular Physiology Cell Physiol Biochem 2019;52:802-821

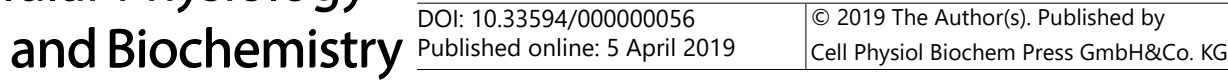 \\ Zehorai et al.: JNK/p38 Translocation is Mediated by Importins3, 7 and 9}

induced phosphorylation of these transcription factors. Interestingly, the strong decrease in transcription factor phosphorylation did not correlate with the medium inhibtion of JNK/p38 translocation $(\sim 60 \%)$ upon Imp7 and Imp9 knockdown. This point was very reproducible accros experiments, and could be explained by the existence of a threshold of MAPK activity that is required for the full activation of the MAPK substrates. Such a threshold has been previously suggested for the action of MAPKs in several systems [38]. No influence of the siRNA of Imp3 and 9 on the ERK target C-Myc, ERK phosphorylation, or RSK were detected in any other proteins examined (data not shown), verifying the specificity of the effects. Thus, it appears that the three Imps are important for the translocation of both JNK1/2 and p38 $\alpha / \beta$, although the role of Imp3 may be more general.

In order to participate in the translocation process, it is possible that unlike $\operatorname{Imp} \alpha / \beta$, Imps 3, 7 and 9 change their localization upon stimulation. Indeed, using fluorescent (Fig. 7A, B) and spinning disk confocal microscopies (Fig. 7C), we found that stimulation of both HeLa and HB2 cells causes a significant shift of Imps7 and Imp9 from the cytoplasm to the nucleus (Imps 7, 9). The localization of Imp3 upon stimulation was changed in a different manner, as some molecules shuttled to the nuclear rim (Fig. 7A), while another part remained in the cytoplasm (mainly in Hela cells). Imp $\beta$ localization was not affected at all upon stimulation. The dynamic changes in Imps 3, 7 and 9 localization were confirmed by nuclear fractionation of stimulated HeLa cells as well (Fig. 7D). These results show for the first time that the $\beta$-like Imps are influenced by extracellular stimulation. It also further confirms that all three Imps are important for the nuclear translocation of JNK1/2 or $\mathrm{p} 38 \alpha / \beta$.

Imps 7/9-JNK/p38 complexes interacts with phosphorylated Imp3

To obtain a better insight into the MAPKs-Imps interactions, we looked into the in vitro interaction of GST-p38 $\alpha / \beta$ and GST-JNK1/2, with purified Imps from extracts of nonstimulated or stimulated Imp-overexpressing HeLa cells. Although the MAPKs interacted with Imps 7 or 9 from stimulated cells as expected, no in vitro interaction was detected with Imp3 (Fig. 8A). Moreover, CoIP experiment revealed that Imp3 is not required for the interaction of JNK or p38 MAPKs with Imp7/9 (Fig. 8B). These results indicate that JNKs and p38s directly interact with either Imp7 or Imp9, but not Imp3, and these interactions do not require Imp3.

The interaction of the MAPKs with Imps 3, 7 and 9 in cells (Fig. 4 and 5) raised the question as to what is the role of each of the Imps in the process of nuclear translocation. The finding that: i) the siRNA of Imp3 had a higher effect than that of Imp7 and Imp9 (Fig. 6), ii) the siRNAs of Imp7 and 9 had an additive effect, iii) Imp3 did not directly interact with JNK1/2 or $\mathrm{p} 38 \alpha / \beta$ in vitro (Fig. 8A), and iv) Imp3 is not required for the MAPKs interactions with Imp7 or Imp9 (Fig. 8B), led to the hypothesis that the MAPKs bind either to Imp7 or Imp9, within heterotrimers of Imp3/Imp7/MAPK or Imp3/Imp9/MAPK. In order to examine this hypothesis, and establish a complex formation between endogenous Imps 3, and 9, we monitored these possible interactions by PLA. No interaction between any of the examined Imps was detected in resting HeLa cells (Fig. 9A, B). However, this was dramatically changed upon stimulation, which induced interactions between Imp3 and either Imp7 or Imp9, but not between Imp7 and Imp9. These results were further confirmed by CoIP experiments of stimulated extracts from HeLa cells as well (Fig. 9C). Interestingly, in vitro interaction of recombinant Imp7 and Imp9 (purified from bacteria) with immunoprecipitated (IPed) Imp3 from non-stimulated or stimulated cells (Fig. 9D), demonstrated that Imp3 is modified upon stimulation to allow the interaction. Treatment of the IPed Imp3 with alkaline phosphatase (CIP) reduced the interaction, strongly indicating that the interaction with either Imp7 or imp9 requires the phosphorylation of Imp3. This figure demonstrates that, as expected, there was no interaction between Imp7 and Imp9. This lack of interaction was not affected by CIP as well. 


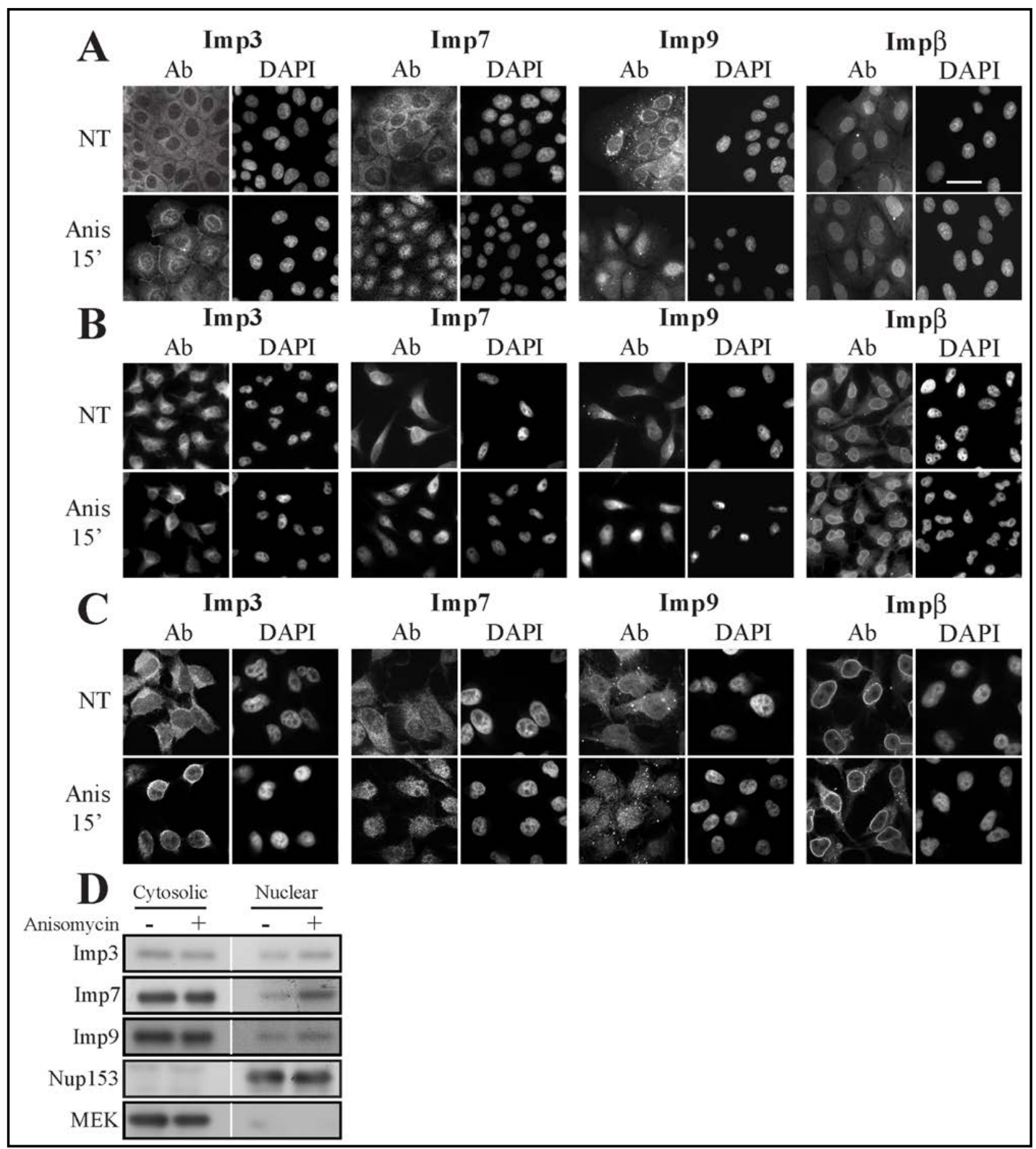

Fig. 7. The subcellular localization of Imps3, 7 and 9 in resting and stimulated cells. Fluorescent microscopy detects the nuclear shuttling of Imp3/7/9. HB2 (A) or HeLa (B, C) cells were grown on slides to $70 \%$ confluence, serum starved, and then either stimulated with anisomycin (Anis $0.5 \mu \mathrm{g} / \mathrm{ml} 15 \mathrm{~min}$ ) or left untreated (NT). Cells were fixed and stained using the indicated Abs. The nuclei were detected using DAPI and slides were visualized using either a fluorescent microscope (A, B) or Zeiss Spinning Disk confocal microscope (C). The bar in the upper right panel of A is of $20 \mu \mathrm{M}$. (D) Subcellular fractionation confirms the nuclear translocation of Imps 3,7 and 9. The same samples of Fig. 1D were used here. Serum-starved HeLa cells were either stimulated (Anis, $0.5 \mu \mathrm{g} / \mathrm{ml}, 15 \mathrm{~min}$ ) or left untreated then harvested. Subcellular fractions of cytosolic and nuclear were produced as described, and subjected to Western blot analysis with the indicated Abs.

\section{Gel filtration analysis of MAPKs-Imps interactions}

To further validate this mechanism, we used gel filtration to separate unbound proteins from higher MW complexes. Indeed, Imps 3, 7 and 9 from resting cells appeared at their expected monomeric MW ( $\sim 90 \mathrm{kDa}$ for Imp3 and $120 \mathrm{kDa}$ for Imp7or Imp9; Fig. 10A). Imp3 also appeared in a $\sim 160 \mathrm{kDa}$ peak, which may represent either a homo or hetero 
Fig. 8. Imp7 and Imp9 but not Imp3 directly interact with JNK/p38 MAPKs. (A) In vitro binding assay demonstrate a direct Imp7or Imp9 interaction with $\mathrm{JNK} 1 / 2$ and $\mathrm{p} 38 \alpha / \beta$. Imps 3,7 and 9 were IPed from either stimulated or untreated cells (NT) and extensively washed with RIPA buffer following LiCl $(0.5 \mathrm{M})$ and buffer A. To examine association, 500 ng of each indicated GSTMAPK were incubated with the indicated IPed Imps ( $2 \mathrm{hr}$ with rotation) following washing and resuspention with sample buffer. Interacting MAPKs were detected using Western blotting with the indicated Abs. (B) Imp3 is not required for Imp7 or Imp9 interactions with MAPKs. SiRNAs of Imp3 and scrambled si of Imp3 that served as control ( $\mathrm{Si}$ SCR) were transfected to HeLa cells, as described. Cell extracts were then subjected to CoIP with the indicated Abs. The amount of CoIPed MAPKs, Imp and input Imps were detected by Western blotting with the indicated Abs. To confirm the efficiency of the siRNA, cells were transfected with
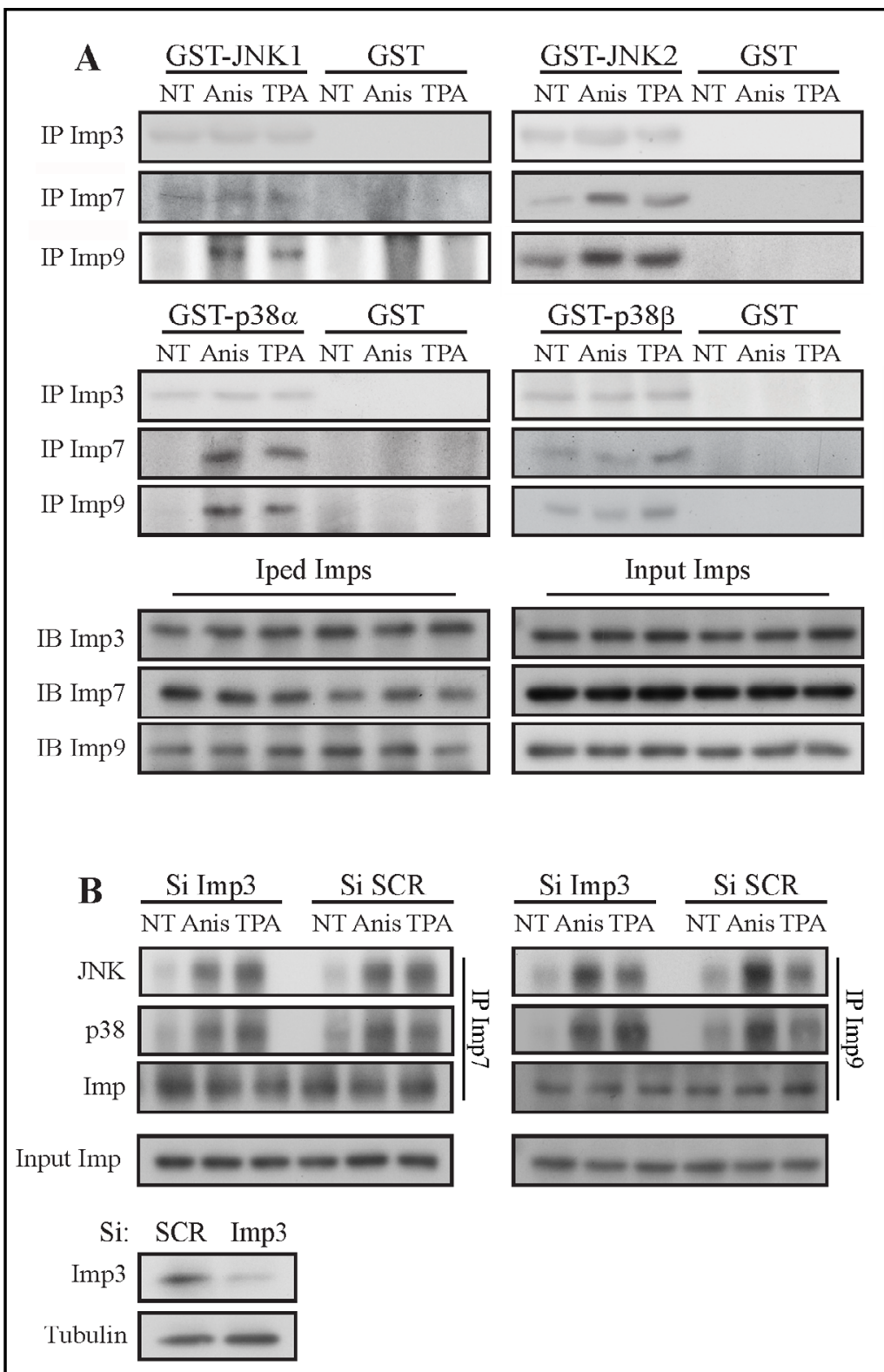

the indicated siRNAs, and then subjected to a Western blot analysis with the indicated Abs (lower panels). The experiments were reproduced three times.

dimer/trimer of the protein. In addition, a small amount of all three non-stimulated Imps was detected in another, $220-400 \mathrm{kDa}$ (designated as $\sim 280 \mathrm{kDa}$ ) peak (Fig. 10A). This relatively wide $\sim 280 \mathrm{kDa}$ peak may correspond to either a dimer of Imps, a heterotrimer containing a dimer of Imps with additional 30-80 kDa proteins (e.g. MAPKs), or a complex of the Imps with any high MW proteins (100-200 kDa). Importantly, the relative amount of the Imps in the various peaks was dramatically changed in extracts from stimulated cells. Thus, the amount of Imps 3, 7 and 9 in the lower MW peaks significantly decreased, while that in the higher ones, correspondingly, increased. In parallel, JNK, and p38 shifted from a sharp peak at $\sim 40 \mathrm{kDa}$, to a very wide peak after stimulation (Fig. 10C). 


\section{Cellular Physiology and Biochemistry

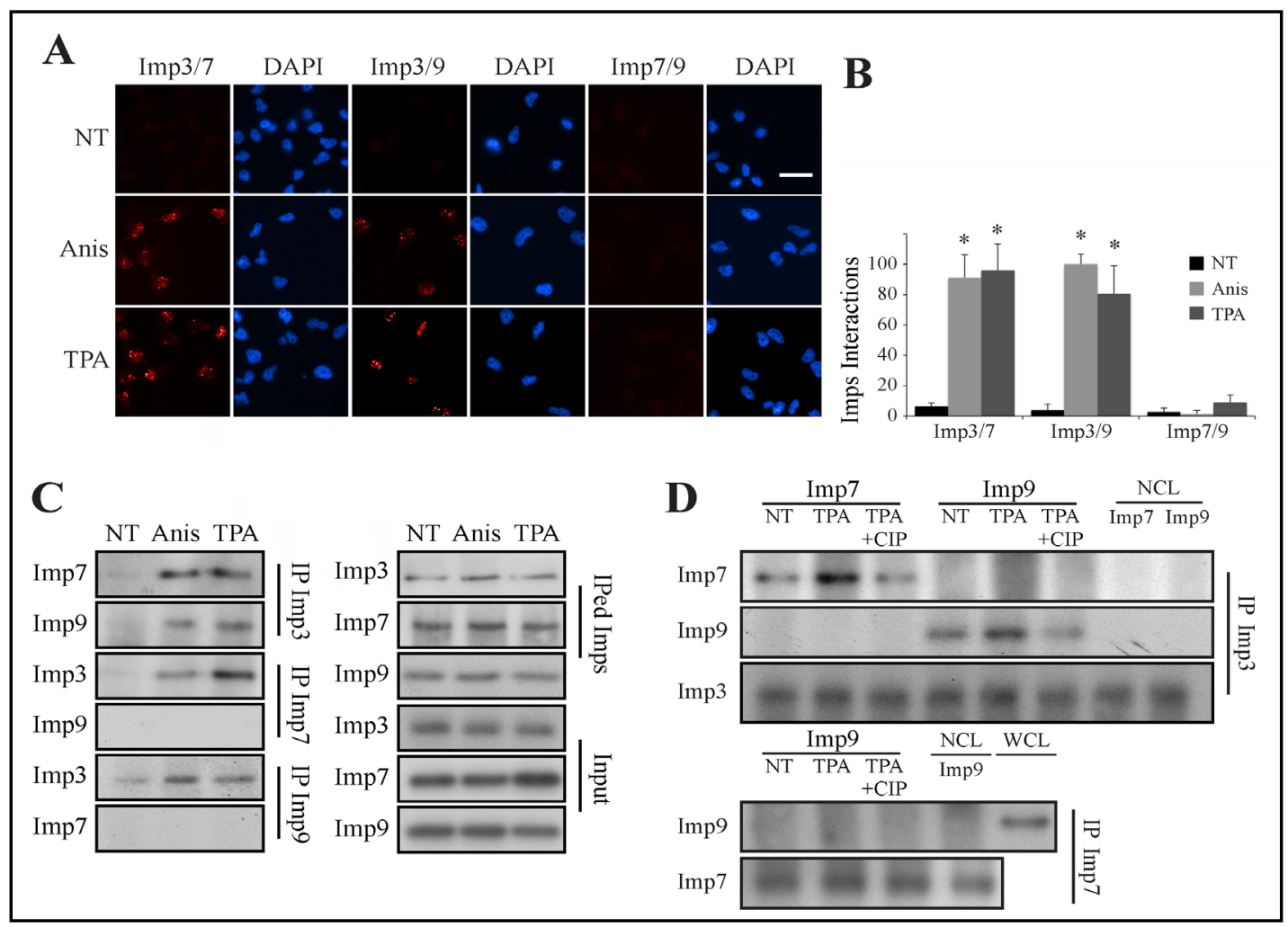

Fig. 9. Imp3 interacts with Imp7/9-MAPK dimer in a phospho-dependent manner. (A) Proximity ligation assay demonstrates stimulus-dependent interaction of Imp3 with Imp7/9. HeLa cells were grown on slides to $70 \%$ confluence, serum starved and either stimulated with anisomycin (Anis, $0.5 \mu \mathrm{g} / \mathrm{ml}, 15$ min) or left untreated (NT). The cells were then fixed and subjected to a PLA using the indicated Abs and visualized using a fluorescent microscope. The bar in the upper right panel is of $20 \mu \mathrm{M}$. (B) Quantification of the intensity of the signals was performed using ImageJ. The data shown represents mean \pm S.E of three different experiments $(*-p<0.0005)$. (C) CoIP assay confirms the interaction of Imp3/7 and Imp3/9. Serumstarved HeLa cells were stimulated with either anisomycin (Anis $0.5 \mu \mathrm{g} / \mathrm{ml}, 15 \mathrm{~min}$ ), TPA (250 nM, $15 \mathrm{~min}$ ) or left untreated (NT). Cells extracts were then subjected to CoIP with indicated Abs. Imps, IPed Imps and inputs were detected by Western blot with the indicated Abs. (D) The interaction of Imp3 with Imp7/9 is dependent on Imp3 phosphorylation. IPed Imp3 (upper panels) or Imp7 (lower panels) were purified from either stimulated or untreated cells, as described. Purified Imps were then incubated with calf intestinal phosphatase, (CIP, $1 \mathrm{hr}, 37^{\circ} \mathrm{C}$ ) or left untreated followed by washing. Recombinant Imps were incubated with the IPed Imps $\left(2 \mathrm{hr}, 37^{\circ} \mathrm{C}\right)$ and then washed. The interacting Imps were detected using Western blotting with the indicated Abs (NCL - no cell lysate, WCL - whole cell lysate). The experiments were reproduced twice.

In order to ascertain that the higher MW peaks of the Imps and MAPKs are formed, at least partially, by interaction between MAPKs and dimers of Imps, we resorted to CoIP experiments. As expected, no association between the components was detected in the $\sim 120$ kDa peaks (Fig. 10B); this was also true for the higher MW peak ( $160 \mathrm{kDa})$ of Imp3 (data not shown). On the other hand, Imps 3, 7 and 9 CoIPed both JNK and p38 proteins from the stimulated, $280 \mathrm{kDa}$ peak. In addition, the Imp3 CoIPed both Imp7 and Imp9, while no interaction between Imp7 and Imp9 was detected. The lack of interaction between Imp7 and Imp9 was observed in higher MW fractions as well, clearly indicating that no Imp3/7/9 trimers are formed after stimulation. No reproducible differences in MAPKs/Imps binding affinity were detected under the distinct stimulations, indicating redundant activities of the dimers with MAPKs. These results further support the stimulated formation of heterotrimers of two Imps with one MAPK, and the role of these heterotrimers for proper translocation to the nucleus. 
A
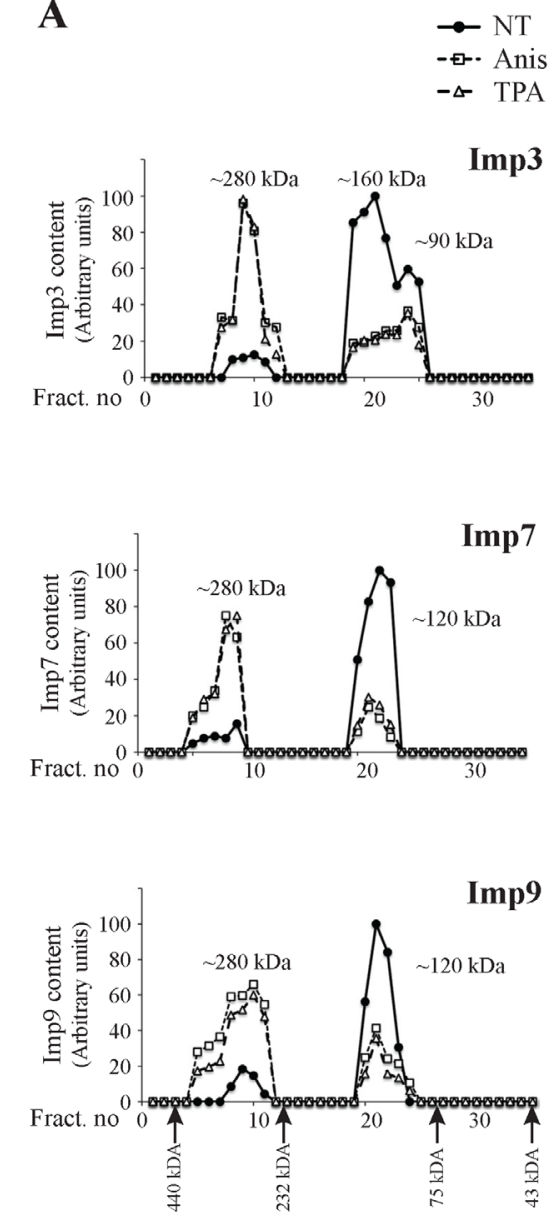
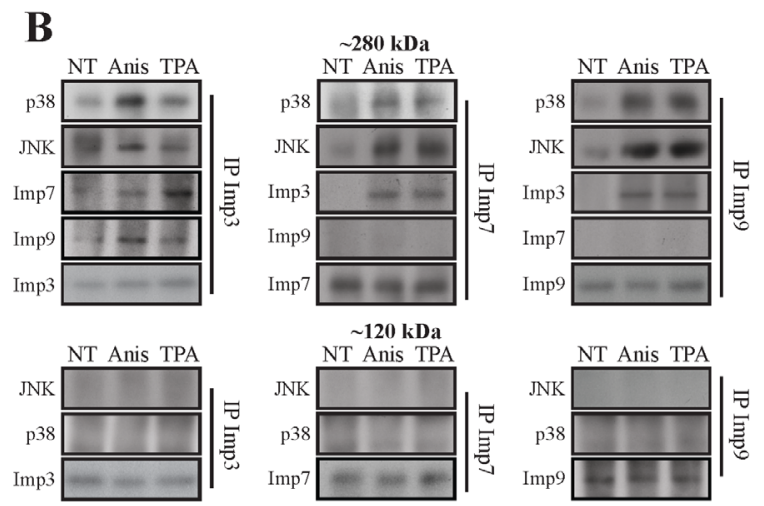

C
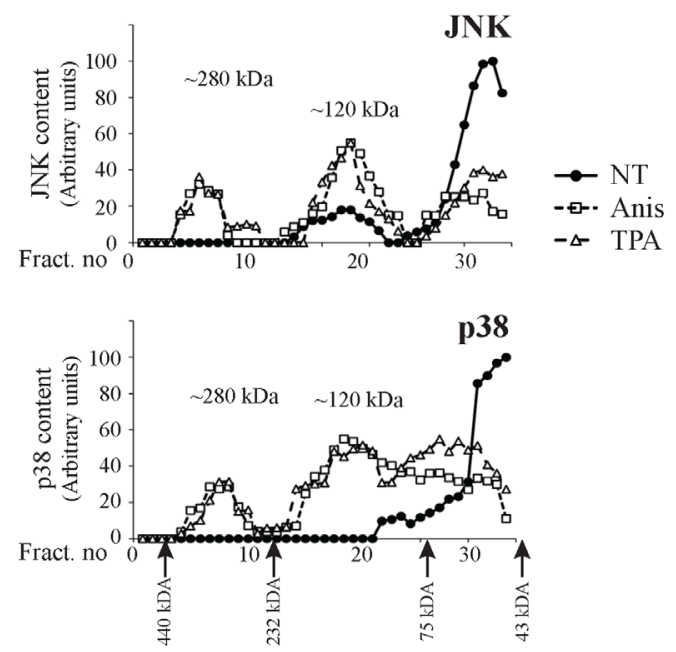

Fig. 10. JNKs and p38s form complexes with dimers of either Imp3/7 or Imp3/9 after stimulation. (A) Gel filtration studies reveal MW shift of Imps 3, 7 and 9 upon stimulation. HeLa cells were serum starved and stimulated with anisomycin, TPA or left untreated (NT). Cell extracts (20 mg) were loaded on a 16/60 superdex 200 sizing column ( $1 \mathrm{ml} / \mathrm{min})$, and $1 \mathrm{ml}$ fractions were collected. The fractions were then analyzed using Western blotting with the indicated Abs. (B) CoIP confirms association of JNKs and p38s with Imps dimers in the $\sim 280 \mathrm{kDa}$ but not the $\sim 120 \mathrm{kDa}$ peaks. Fractions representing the $\sim 280 \mathrm{kDa}$ and $\sim 120 \mathrm{kDa}$ peaks (fractions no. 9 and 22) from each of the Anis, TPA or non- treated stimulated columns, were subjected to CoIP with the indicated Imps Abs. The interacting proteins were detected using Western blotting with the indicated Abs. (C) JNK and p38 MAPKs form complexes upon stimulation. HeLa cells were serum starved and then stimulated with anisomycin, TPA or left untreated (NT). Cell extracts were loaded on a 16/60 superdex 200 sizing column (flow rate $1 \mathrm{ml} / \mathrm{min}$ ), and $1 \mathrm{ml}$ fractions were collected. The fractions were then analyzed, using Western blot with the anti JNK/p38 Abs, as indicated.

\section{Discussion}

Stimulation of cells by various extracellular agents induces changes in the localization of many signaling proteins into the nucleus (translocation), a step important for the regulation of transcription and induction of various cellular processes. Indeed, we have recently shown that prevention of the translocation of these MAPKs leads to a reduced proliferation of triple-negative breast cancer cells, reduced DSS-induced inflammation in mice, and reduced inflammation-related colon cancer in mice [25]. Despite the importance of the 
stimulated translocation, the molecular mechanism that allows it, is not yet clear. Here, we studied the stimulated nuclear translocation of JNK1/2 and $\mathrm{p} 38 \alpha / \beta$, and found that all four kinases rapidly translocate to the nucleus upon stress and mitogenic stimuli. However, the molecular mechanism of these translocations seems to be distinct from that of ERK1/2 [8], or any other known translocation mechanisms $[37,39,40]$. Rather, the results presented here best fit a model in which upon stimulation, JNK1/2 and p38 $\alpha / \beta$ are released from their cytoplasmic anchors by an activation-independent mechanism. This allows the formation of heterotrimers that include either active or inactive MAPKs, post-translationaly modified Imp7 or Imp9, and phosphorylated Imp3. The Imp3/Imp7 or Imp3/Imp9 dimers then escort the attached MAPKs to the nuclear envelope, where Imp3 remains, while Imp7/9 further penetrate into the nucleus together with the MAPKs (see scheme in [41]). Then, the complex is dissociated by GTP-Ran, which frees the MAPKs in the nucleus and allows the export of the Imps back to the cytosol.

MAPKs are just a small portion of the many proteins that translocate to the nucleus upon various extracellular stimulations. Thus, any stimuli induces a swift nuclear translocation of tens of millions of protein molecules, that are primarily required for the induction of transcription, and as a consequence, various cellular processes. This large scale and rapid translocation requires a handy and robust translocating machinary. Indeed, some of the proteins seem to use the canonical NLS-Imp $\alpha / \beta$ for their translocation (e.g. NFkB [3], or ERK5 [5]). However, the limited number of Imp $\alpha$ molecules, and the fact that most of them are preoccupied by housekeeping shuttling proteins [42], indicate that there are not sufficient free Imp $\alpha$ molecules to carry out the rapid and massive translocation upon stimulation. In addition, the rapid nature of the process, as well as the size of most translocating proteins, do not allow a free diffusion or carrier-free penetration through NUPs interactions [39]. This suggests the action of stimulated, NLS- and diffusion-independent, translocation machineries. Indeed, our results do not show any interaction with either Imp $\alpha$ or $\operatorname{Imp} \beta$ under the conditions used. However, a recent article has reported such interaction with Imps $\alpha 2 / \beta 1$ upon stress [43]. The reason for these discrepancy is not clear, but may occur due to distinct cell lines and stimulations used.

Our data here, as well as previous publications [12], strongly indicate that $\beta$-like Imps play key roles in NLS- and Imp $\alpha$-independent mechanisms. Despite the clear role of Imps 3, 7 and 9 in stimulation-dependent translocation shown here, previous studies demonstrated that these Imps induce nuclear shuttling of non-stimulated proteins as well. Therefore, it is likely that $\beta$-like Imps are able to mediate nuclear shuttling by more than one mechanism. An example for multiple mechanisms of action is demonstrated by Imp7, which induces shuttling of various proteins in both resting and stimulated cells. In resting cells, Imp7 appears to induce the nuclear shuttling of housekeeping (e.g. histones [44, 45], and ribosomal proteins [46]), as well as regulatory proteins (e.g. EZI [47], GR [48], and CDK5 activator [49]). Although Imp7 may act on its own, some reports demonstrated the requirement of its heterodimerization with $\operatorname{Imp} \beta[50,51]$. In all of the above cases, it was either shown, or speculated, that Imp7 binds canonical NLS or NLS-like sequences in their cargo. Interestingly, Imp7 also mediates the stimulated translocation of several signaling proteins (e.g. ERK1/2, MEK1/2, Smad3/4, EGR1) operating in a canonical NLS-independent manner by binding to specific sequences (e.g. NTS of ERK1/2) in its cargo [7, 8, 11, 52]. Here we show, yet another canonical NLSindependent mechanism for the stimuli-dependent nuclear translocation of JNK and p38 by Imp7. Thus, Imp7 is involved in multiple mechanisms and interactions with various cargo sequences to induce both stimulated and non-stimulated nuclear translocation of proteins. We also found that Imp7 undergoes a stimuli-induced modification to allow its binding to JNK and p38 MAPKs. Therefore, it is likely that the switch from one mechanism to another is mediated by post-translational modifications such as phosphorylation, ubiquitination or sulfation. The large variety of mechanisms by which Imp7 and the other $\beta$-like Imps operate may contribute to their specificity in regulating distinct processes upon different stimuli and conditions. 


\section{Cellular Physiology Cell Physiol Biochem 2019;52:802-821

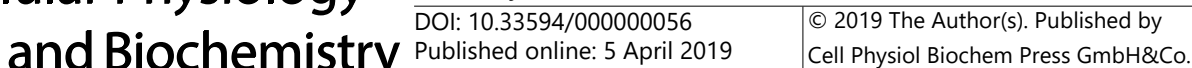 \\ Zehorai et al.: JNK/p38 Translocation is Mediated by Importins3, 7 and 9}

\section{Conclusion}

In summary, we identified a novel mechanism of stimulated nuclear translocation of active/inactive JNK/p38 MAPKs, which is distinct from the stimulated translocation of ERK1/2. We show that this translocation involves binding of these MAPKs to either Imp7 or Imp9, which operate in conjugation with Imp3, in order to allow the nuclear translocation of the MAPKs. This is the first demonstration of dimerization of the $\beta$-like Imps with Imps other than $\operatorname{Imp} \beta$ itself. Unlike Imp $\alpha / \beta$, these heterotrimers then translocate to the perinuclear region, where they dissociate; Imp3 remains in the nuclear envelope, while the MAPKs, together with either Imp7 or Imp9, shuttle into the nucleus, in a Ran-dependent manner. This study clearly demonstrates that $\beta$-like Imps are central mediators of the stimulated nuclear translocation of JNK/p38 and the activation of transcription factors. Therefore, these components and the process of nuclear translocation are essential for the regulation of stimulated transcription and gene expression.

\section{Acknowledgements}

We would like to thank Tamar Kreizman, Tamar Hanoch, Prof. Oded Livnah, Dr. Galia Maik-Rachline and Lior Abramson for their help. Supported by grants from the Israeli Science Foundation and Minerva. RS is an incumbent of the Yale S. Lewine and Ella Miller Lewine professorial chair for cancer research.

\section{Disclosure Statement}

The authors report no conflict of interest.

\section{References}

- 1 Plotnikov A, Zehorai E, Procaccia S, Seger R: The MAPK cascades: Signaling components, nuclear roles and mechanisms of nuclear translocation. Biochim Biophys Acta 2011;1813:1619-1633.

- 2 Wainstein E, Seger R: The dynamic subcellular localization of ERK: mechanisms of translocation and role in various organelles. Curr Opin Cell Biol 2016;39:15-20.

- 3 Magnani M, Crinelli R, Bianchi M, Antonelli A: The ubiquitin-dependent proteolytic system and other potential targets for the modulation of nuclear factor-kB (NF-kB). Curr Drug Targets 2000;1:387-399. Gorlich D: Nuclear protein import. Curr Opin Cell Biol 1997;9:412-419.

$>4$ Kondoh K, Terasawa K, Morimoto H, Nishida E: Regulation of nuclear translocation of extracellular signalregulated kinase 5 by active nuclear import and export mechanisms. Mol Cell Biol 2006;26:1679-1690.

- 6 Fagotto F, Gluck U, Gumbiner BM: Nuclear localization signal-independent and importin/karyopherinindependent nuclear import of beta-catenin. Curr Biol 1998;8:181-190.

7 Yao X, Chen X, Cottonham C, Xu L: Preferential utilization of Imp7/8 in nuclear import of Smads. J Biol Chem 2008;283:22867-22874.

8 Chuderland D, Konson A, Seger R: Identification and characterization of a general nuclear translocation signal in signaling proteins. Mol Cell 2008;31:850-861.

9 Plotnikov A, Chuderland D, Karamansha Y, Livnah O, Seger R: Nuclear ERK translocation is mediated by protein kinase CK2 and accelerated by autophosphorylation. Mol Cell Biol 2011;31:3515-3530.

- 10 Plotnikov A, Flores K, Maik-Rachline G, Zehorai E, Kapri-Pardes E, Berti DA, Hanoch T, Besser MJ, Seger R: The nuclear translocation of ERK1/2 as an anticancer target. Nat Commun 2015;6:6685.

- 11 Chen J, Liu MY, Parish CR, Chong BH, Khachigian L: Nuclear import of early growth response-1 involves importin-7 and the novel nuclear localization signal serine-proline-serine. Int J Biochem Cell Biol 2011;43:905-912. 


\section{Cellular Physiology Cell Physiol Biochem 2019;52:802-821

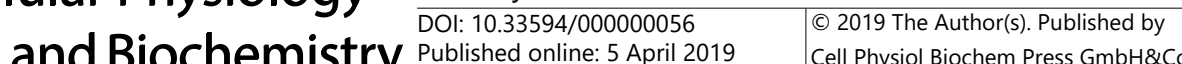 and Biochemistry Published online: 5 April 2019 Cell Physiol Biochem Press GmbH\&Co. KG \\ Zehorai et al.: JNK/p38 Translocation is Mediated by Importins3, 7 and 9}

- 12 Chook YM, Suel KE: Nuclear import by karyopherin-betas: recognition and inhibition. Biochim Biophys Acta 2011;1813:1593-1606.

- 13 Flores K, Seger R: Stimulated nuclear import by $\beta$-like importins. F1000Prime Reports 2013;5:41.

- 14 Joseph J: Ran at a glance. J Cell Sci 2006;119:3481-3484.

- 15 Waldmann I, Walde S, Kehlenbach RH: Nuclear import of c-Jun is mediated by multiple transport receptors. J Biol Chem 2007;282:27685-27692.

- 16 Chachami G, Paraskeva E, Georgatsou E, Bonanou S, Simos G: Bacterially produced human HIF-1alpha is competent for heterodimerization and specific DNA-binding. Biochem Biophys Res Commun 2005;331:464-470.

- 17 Keshet Y, Seger R: The MAP kinase signaling cascades: a system of hundreds of components regulates a diverse array of physiological functions. Methods Mol Biol 2010;661:3-38.

- 18 Nishimoto S, Nishida E: MAPK signalling: ERK5 versus ERK1/2. EMBO Rep 2006;7:782-786.

- 19 Morrison DK, Davis RJ: Regulation of MAP kinase signaling modules by scaffold proteins in mammals. Annu Rev Cell Dev Biol 2003;19:91-118.

- 20 Whitmarsh AJ: The JIP family of MAPK scaffold proteins. Biochem Soc Trans 2006;34:828-832.

- 21 Cavigelli M, Dolfi F, Claret FX, Karin M: Induction of c-fos expression through JNK-mediated TCF/Elk-1 phosphorylation. EMBO J 1995;14:5957-5964.

- 22 Zhang S, Lin Y, Kim YS, Hande MP, Liu ZG, Shen HM: c-Jun N-terminal kinase mediates hydrogen peroxide-induced cell death via sustained poly(ADP-ribose) polymerase-1 activation. Cell Death Differ 2007;14:1001-1010.

- 23 Brand P, Plochmann S, Valk E, Zahn S, Saloga J, Knop J, Becker D: Activation and translocation of p38 mitogen-activated protein kinase after stimulation of monocytes with contact sensitizers. J Invest Dermatol 2002;119:99-106.

24 Wood CD, Thornton TM, Sabio G, Davis RA, Rincon M: Nuclear localization of p38 MAPK in response to DNA damage. Int J Biol Sci 2009;5:428-437.

25 Maik-Rachline G, Zehorai E, Hanoch T, Blenis J, Seger R: The nuclear translocation of the kinases p38 and JNK promotes inflammation-induced cancer. Sci Signal 2018;11:pii:eaao3428.

26 Guo L, Guo Y, Xiao S, Shi X: Protein kinase p-JNK is correlated with the activation of AP-1 and its associated Jun family proteins in hepatocellular carcinoma. Life Sci 2005;77:1869-1878.

27 Cui J, Wang Q, Wang J, Lv M, Zhu N, Li Y, Feng J, Shen B, Zhang J: Basal c-Jun NH2-terminal protein kinase activity is essential for survival and proliferation of T-cell acute lymphoblastic leukemia cells. Mol Cancer Ther 2009;8:3214-3222.

- 28 Lee SH, Park J, Che Y, Han PL, Lee JK: Constitutive activity and differential localization of p38alpha and p38beta MAPKs in adult mouse brain. J Neurosci Res 2000;60:623-631.

- 29 Posen Y, Kalchenko V, Seger R, Brandis A, Scherz A, Salomon Y: Manipulation of redox signaling in mammalian cells enabled by controlled photogeneration of reactive oxygen species. J Cell Sci 2005;118:1957-1969.

30 Girnius N, Edwards YJ, Garlick DS, Davis RJ: The cJUN NH2-terminal kinase (JNK) signaling pathway promotes genome stability and prevents tumor initiation. Elife 2018;7:pii:e36389.

31 Pei H, Zhang J, Nie J, Ding N, Hu W, Hua J, Hirayama R, Furusawa Y, Liu C, Li B, Hei TK, Zhou G: RAC2-P38 MAPK-dependent NADPH oxidase activity is associated with the resistance of quiescent cells to ionizing radiation. Cell Cycle 2017;16:113-122.

32 Ben-Levy R, Hooper S, Wilson R, Paterson HF, Marshall CJ: Nuclear export of the stress-activated protein kinase p38 mediated by its substrate MAPKAP kinase-2. Curr Biol 1998;8:1049-1057.

33 Boussif O, Lezoualc'h F, Zanta MA, Mergny MD, Scherman D, Demeneix B, Behr JP: A versatile vector for gene and oligonucleotide transfer into cells in culture and in vivo: polyethylenimine. Proc Natl Acad Sci U S A 1995;92:7297-7301.

- 34 Lowder MA, Appelbaum JS, Hobert EM, Schepartz A: Visualizing protein partnerships in living cells and organisms. Curr Opin Chem Biol 2011;15:781-788.

- 35 Marfori M, Mynott A, Ellis JJ, Mehdi AM, Saunders NF, Curmi PM, Forwood JK, Boden M, Kobe B: Molecular basis for specificity of nuclear import and prediction of nuclear localization. Biochim Biophys Acta 2011;1813:1562-1577.

- 36 Lange A, Mills RE, Lange CJ, Stewart M, Devine SE, Corbett AH: Classical nuclear localization signals: definition, function, and interaction with importin alpha. J Biol Chem 2007;282:5101-5105. 


\section{\begin{tabular}{lll} 
Cellular Physiology & Cell Physiol Biochem 2019;52:802-821 \\
\cline { 2 - 2 } & DOl: 10.33594/000000056 & O 2019 The Author(s). Published by
\end{tabular} \begin{tabular}{l|l} 
and Biochemistry Poblished online: 5 April 2019 & $\begin{array}{l}\text { De } 2019 \text { The Author(s). Published by } \\
\text { Cell Physiol Biochem Press GmbH\&Co. KG }\end{array}$ \\
\hline
\end{tabular} \\ Zehorai et al.: JNK/p38 Translocation is Mediated by Importins3, 7 and 9}

37 Christophe D, Christophe-Hobertus C, Pichon B: Nuclear targeting of proteins: how many different signals? Cell Signal 2000;12:337-341.

- 38 Matheny SA, Chen C, Kortum RL, Razidlo GL, Lewis RE, White MA: Ras regulates assembly of mitogenic signalling complexes through the effector protein IMP. Nature 2004;427:256-260.

- 39 Xu L, Massague J: Nucleocytoplasmic shuttling of signal transducers. Nat Rev Mol Cell Biol 2004;5:209-219.

- 40 Gorlich D, Kutay U: Transport between the cell nucleus and the cytoplasm. Annu Rev Cell Dev Biol 1999;15:607-660.

- 41 Flores K, Katz AA, Yadav SS, Seger R: The nuclear translocation of mitogen-activated protein kinases: molecular mechanisms and use as novel therapeutic target. Neuroendocrinology 2019;108:121-131.

42 Nevo R, Stroh C, Kienberger F, Kaftan D, Brumfeld V, Elbaum M, Reich Z, Hinterdorfer P: A molecular switch between alternative conformational states in the complex of Ran and importin beta1. Nat Struct Biol 2003;10:553-557.

43 Misheva M, Kaur G, Ngoei KR, Yeap YY, Ng IH, Wagstaff KM, Ng DC, Jans DA, Bogoyevitch MA: Intracellular mobility and nuclear trafficking of the stress-activated kinase JNK1 are impeded by hyperosmotic stress. Biochim Biophys Acta 2014;1843:253-264.

44 Muhlhausser P, Muller EC, Otto A, Kutay U: Multiple pathways contribute to nuclear import of core histones. EMBO Rep 2001;2:690-696.

- 45 Baake M, Bauerle M, Doenecke D, Albig W: Core histones and linker histones are imported into the nucleus by different pathways. Eur J Cell Biol 2001;80:669-677.

- 46 Jakel S, Gorlich D: Importin beta, transportin, RanBP5 and RanBP7 mediate nuclear import of ribosomal proteins in mammalian cells. EMBO J 1998;17:4491-4502.

- 47 Saijou E, Itoh T, Kim KW, Iemura S, Natsume T, Miyajima A: Nucleocytoplasmic shuttling of the zinc finger protein EZI Is mediated by importin-7-dependent nuclear import and CRM1-independent export mechanisms. J Biol Chem 2007;282:32327-32337.

- 48 Freedman ND, Yamamoto KR: Importin 7 and importin alpha/importin beta are nuclear import receptors for the glucocorticoid receptor. Mol Biol Cell 2004;15:2276-2286.

49 Fu X, Choi YK, Qu D, Yu Y, Cheung NS, Qi RZ: Identification of nuclear import mechanisms for the neuronal Cdk5 activator. J Biol Chem 2006;281:39014-39021.

50 Jakel S, Mingot JM, Schwarzmaier P, Hartmann E, Gorlich D: Importins fulfil a dual function as nuclear import receptors and cytoplasmic chaperones for exposed basic domains. EMBO J 2002;21:377-386.

51 Bauerle M, Doenecke D, Albig W: The requirement of H1 histones for a heterodimeric nuclear import receptor. J Biol Chem 2002;277:32480-32489.

52 Xu L, Alarcon C, Col S, Massague J: Distinct domain utilization by Smad3 and Smad4 for nucleoporin interaction and nuclear import. J Biol Chem 2003;278:42569-42577. 